\title{
EQUIVALENCE CLASSES OF MATRICES OVER A FINITE FIELD
}

\author{
GARY L. MULLEN \\ Department of Mathematics \\ The Pennsylvania State University \\ Sharon, Pennsylvania 16146 \\ (Received October 26, 1978)
}

ABSTRACT. Let $F_{q}=G F(q)$ denote the finite field of order $q$ and $F(m, q)$ the ring of mxm matrices over $F_{q}$. Let $\Omega$ be a group of permutations of $F_{q}$. If $A, B \in F(m, q)$ then $A$ is equivalent to $B$ relative to $\Omega$ if there exists $\phi \varepsilon \Omega$ such that $\phi(A)=B$ where $\phi(A)$ is computed by substitution. Formulas are given for the number of equivalence classes of a given order and for the total number of classes induced by a cyclic group of permutations.

KEY WORD AND PHRASES. Equivalence, permutation, automorphism, finite field. 1980 MATHEMATICS SUBJECT CLASSIFICATION CODES. Primary 15A33; Secondary $15 A 24$.

1. INTRODUCTION.

In the series of papers [2], [4], [8], and [9], L. Carlitz, S. Cavior, and the author studied several forms of equivalence of functions over a finite field. In this paper we study a form of equivalence defined on the ring of mxm matrices over a finite field. 
Let $F_{q}=G F(q)$ denote the finite field of order $q$ and $F(m, q)$ the ring of mxm matrices over $F_{q}$. Let $\Omega$ be a group of permutations on $F_{q}$ so that $\Omega$ Is isomorphic to a subgroup of $\mathrm{S}_{\mathrm{q}}$, the symmetric group on $\mathrm{q}$ letters.

2. GENERAL THEORY.

A permutation $\phi$ of $\mathrm{F}_{\mathrm{q}}$ is a function from $\mathrm{F}_{\mathrm{q}}$ onto $\mathrm{F}_{\mathrm{q}}$ and so by the Lagrange Interpolation Formula $[6, \mathrm{p} .55]$, is expressible as a polynomial of degree less than $q$. Suppose $\phi(x)=a_{n} x^{n}+\ldots+a_{1} x+a_{0}$ where each $a_{1} \in F_{q}$ and $\mathrm{n} \leq \mathrm{q}-1$. Then by substitution we may compute the $\operatorname{mxm} \operatorname{matrix} \phi(A)=$ $a_{n} A^{n}+\ldots+a_{1} A+a_{o} I_{m}$ where $I_{m}$ is the $m x m$ identity matrix. If $\Omega$ is a group of permutations then we can make

DEFINITION 1. If $A, B \in F(m, q)$ then $A$ is equivalent to $B$ relative to $\Omega$ if $B=\phi(A)$ for some $\phi \varepsilon \Omega$.

As this relation is an equivalence relation on $F(m, q)$ we let $\mu(A, \Omega)$ denote the order of the class of A relative to $\Omega$ and $\lambda(\Omega)$ denote the number of classes induced by $\Omega$.

DEFINITION 2. If $A \varepsilon F(m, q)$ then a permutation $\phi \varepsilon \Omega$ is an automorphism of $A$ relative to $\Omega$ if $\phi(A)=A$.

Let Aut $(A, \Omega)$ and $v(A, \Omega)$ denote the group and number of automorphisms of the matrix $A$ relative to $\Omega$. If $\phi(A)=B$ for some $\phi \varepsilon \Omega$ then

$$
\operatorname{Aut}(B, \Omega)=\phi \operatorname{Aut}(A, \Omega) \phi^{-1}
$$

so that $v(B, \Omega)=v(A, \Omega)$. Thus the number of automorphisms depends only upon the class and not on the particular matrices within the class. One can now easily prove:

THEOREM 2.1. Let $A \in F(m, q)$. Then for any group $\Omega$

$$
\mu(A, \Omega) v(A, \Omega)=|\Omega|
$$

where $|\Omega|$ denotes the order of the group $\Omega$.

If $\phi$ is a permutation let $I(\phi, m)$ denote the number of mxm matrices $A$ 
such that $\phi(A)=0$. Suppose $\phi(x)=a_{n} x^{n}+\ldots+a_{1} x+a_{0}$ where each $a_{i} \varepsilon F_{q}$ and $a_{n} \neq 0$. Then if $\phi(A)=A$ we have the matrix equation

$$
A^{n}+\frac{a_{n-1} A^{n-1}}{a_{n}}+\ldots+\frac{\left(a_{1}-1\right)}{a_{n}} A+\frac{a_{0}}{a_{n}} I_{m}=0 .
$$

Consider the monic polynomial

$$
E_{\phi}(x)=x^{n}+\frac{a_{n-1}}{a_{n}} x^{n-1}+\ldots+\frac{\left(a_{1}-1\right)}{a_{n}} x+\frac{a_{0}}{a_{n}}
$$

over GF( $q)$. Hodges in [7] gives a formula for the number $N\left(E_{\phi}, m\right)$ of $m \times m$ matrices $A$ over $G F(q)$ such that $E_{\phi}(A)=0$. Clearly $I(\phi, m)=N\left(E_{\phi}, m\right)$ and moreover this number can be computed as follows.

Berlekamp in [1] has given an algorithm for factoring polynomials over $F_{q}$ in terms of solving a system of linear equations over $F_{q}$. Suppose $E_{\phi}$ has the prime factorization $E_{\phi}=P_{1}^{h_{1}} \ldots P_{s}^{h}$ where the $P_{i}^{\prime}$ 's are distinct monic polynomials, $h_{1} \geq 1$ and the degree of $P_{i}$ is $d_{i}$ for $i=1, \ldots, s$. Let $g(t, d)$ represent the number of non-singular matrices of order $t \geq 1$ over $G F\left(q^{d}\right)$ so that, as is well known, $g(t, d)=\prod_{i=0}^{t-1}\left(q^{d t}-q^{d i}\right)$. We also set $g(0, d)=1$.

Let $\pi$ be a partition of $m$ defined by

$$
m=\sum_{i=1}^{s} d_{i} \sum_{j=1}^{h} j k_{i j}, k_{i j} \geq 0 .
$$

For $1=1, \ldots, s$ let

$$
b_{i}(\pi)=\sum_{u=1}^{h_{1}}\left[k_{i u}^{2}(u-1)+2 u k_{i u} \sum_{v=u+1}^{h_{i}} k_{i v}\right]
$$

and set $a(\pi)=\sum_{i=1}^{s} d_{i} b_{i}(\pi)$. Then as shown by Hodges in [7].

$$
I(\phi, m)=N\left(E_{\phi}, m\right)=g(m, 1) \sum_{\pi} g^{-a(\pi)} \prod_{i=1}^{s} \prod_{j=1}^{h_{i}} g\left(k_{1 j}, d_{i}\right)^{-1}
$$

where the sum is over all partitions $\pi$ of $m$ given by (2.5).

3. CYCLIC GROUPS.

Let $\Omega$ be a cyclic group of order $n$, say $\Omega=\langle\phi\rangle$, and let $H(t)$ denote 
the subgroup of $\Omega$ of order $t$, where $t \mid n$. It follows that $H(t)=\left\langle\phi^{n} \mid t\right\rangle$. Let $M(t, m)$ denote the number of $m \times m$ matrices $A$ such that Aut $(A, \Omega)=H(t)$. THEOREM 3.1. For each divisor $t$ of $n$

$$
M(t, m)=N\left(E_{\phi^{n}} n / t, m\right)-\sum N\left(E_{\phi^{n} / u}, m\right)
$$

where the sum is over all $u$ for which $u|n, t| u$, and $t \neq u$.

PROOF. The number of $m \times m$ matrices $A$ such that $H(t) \leq \operatorname{Aut}(A, \Omega)$ is given by $N\left(E \phi^{n / t}, m\right)$. From this we subtract those for which the containment if proper.

COROLLARY 3.2. For all $t / n$ there are $t M(t, m) / n$ classes of order $n / t$ and

$$
\lambda(\Omega)=\frac{1}{n} \sum_{t \mid n} \operatorname{tM}(t, m) .
$$

Following Definition 3.1 of [8] we say that two groups $\Omega_{1}$ and $\Omega_{2}$ induce equivalent decompositions of $F(m, q)$ is they induce the same number of classes of the same size. It is easy to see that if $\Omega=\left\langle\phi_{1}\right\rangle$ and $\Omega_{2}=\left\langle\phi_{2}\right\rangle$ are cyclic groups of order $n$ then $\Omega_{1}$ and $\Omega_{2}$ will induce equivalent decompositions of $F(m, q)$ if for each $t \mid n, \phi_{1}^{n / t}$ and $\phi_{2}^{n / t}$ have the same number of distinct prime factors of the same degree and multiplicity.

As a simple illustration of the above theory let $m=2$ and consider $\phi(x)=x^{3}$ over $G F(5)$ so that if $\Omega=\langle\phi\rangle$ then $|\Omega|=2$. We have $E_{\phi}(x)=x^{3}-x=x(x-1)(x+1)$ so that $s=3, P_{1}=x, P_{2}=x-1, P_{3}=x+1$ and $h_{i}=d_{i}=1$ for $i=1,2,3$. There are six partitions defined by (2.5) which are given by $2=k_{11}+k_{21}+k_{31}$ where $0 \leq k_{1 j} \leq 2$. Clearly $b_{1}(\pi)=0$ for $i=1,2,3$ so that $a(\pi)=0$ for each partition $\pi$. One checks that $g(2,1)=$ $\left(q^{2}-1\right)\left(q^{2}-q\right)=480, g(1,1)=q-1=4$ and $g(0,1)=1$. Hence using $(2.7)$ it is not difficult to check that $N\left(E_{\phi}, 2\right)=93$ so that by $(3.1), M(2,2)=93$ and thus $M(1,2)=532$. Hence by Corollary 3.2 there are 93 classes of order one and 266 classes of order two so that by (3.2) $\lambda(\Omega)=359$. 


\section{REFERENCES}

1. Berlekamp, E. R., "Factoring polynomials over finite flelds," Bell System Technical J. 46 (1967), 1853-1859.

2. Carlitz, L., "Invariantive theory of equations in a finite field," Trans. Amer. Math. Soc. 75 (1953), 405-427.

3. Carlitz, L., "Invariant theory of systems of equations in a finite field," J. Analyse Math. $3(1953-54), 382-413$.

4. Cavior, S. R., "Equivalence classes of functions over a finite field," Acta Arith. 10 (1964), 119-136.

5. Cavior, S. R., "Equivalence classes of sets of polynomials over a finite field," Journ. Reine Angew. Math. 225 (1967), 191-202.

6. Dickson, L. E., Linear Groups with an Exposition of the Galois Field Theory, Dover Publications, Inc., New York, 1958.

7. Hodges, J. H., "Scalar polynomials equations for matrices over a finite field," Duke Math. J. 25 (1958), 291-296.

8. Mullen, G. L., "Equivalence classes of functions over a finite fleld," Acta Arith. 29 (1976), 353-358.

9. Mullen, G. L., "Equivalence classes of polynomials over finite fields," Acta Arith. 31 (1976), 113-123.

10. Mullen, G. L., "Equivalence classes of sets of polynomials over finite fields," To appear, Bull. Calcutta Math. Soc. 


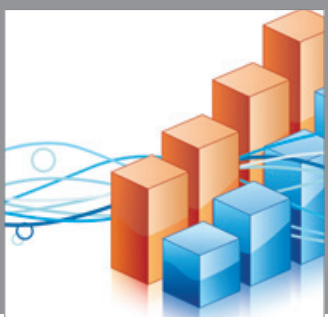

Advances in

Operations Research

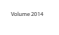

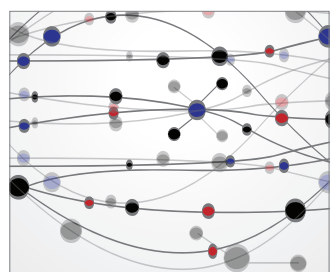

\section{The Scientific} World Journal
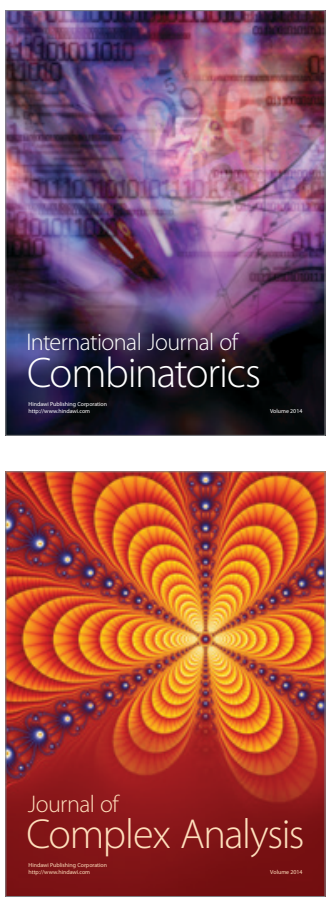

International Journal of

Mathematics and

Mathematical

Sciences
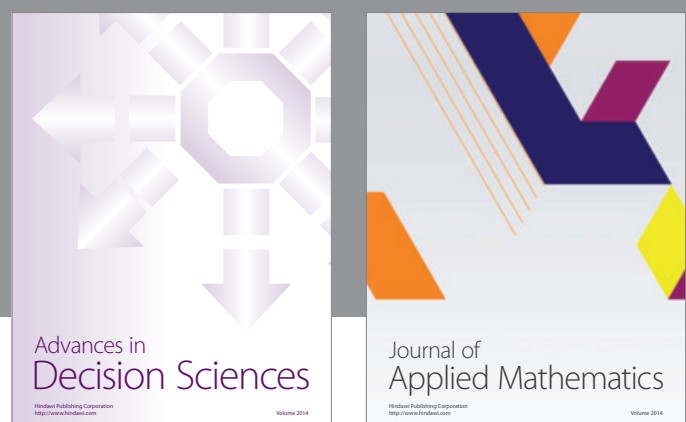

Journal of

Applied Mathematics
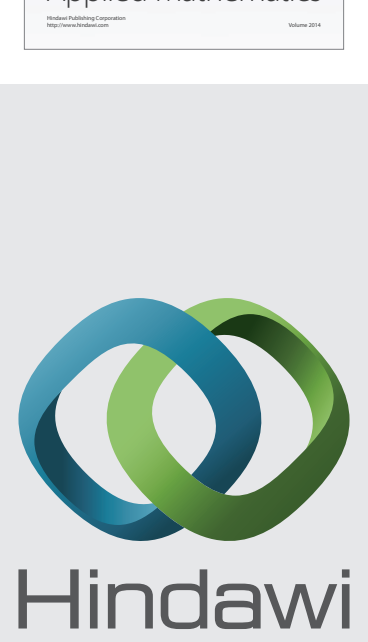

Submit your manuscripts at http://www.hindawi.com
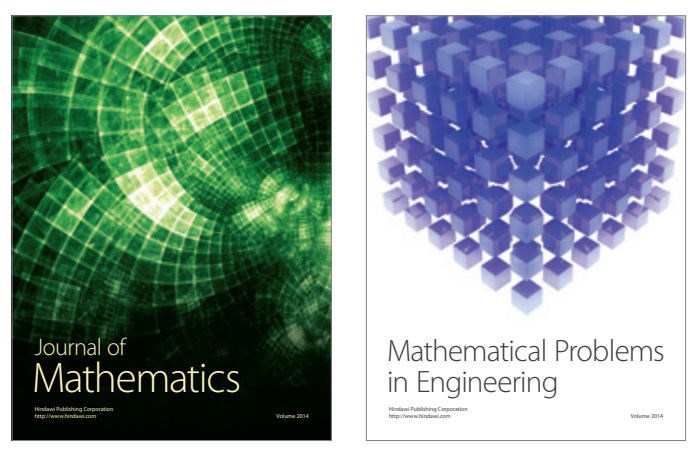

Mathematical Problems in Engineering
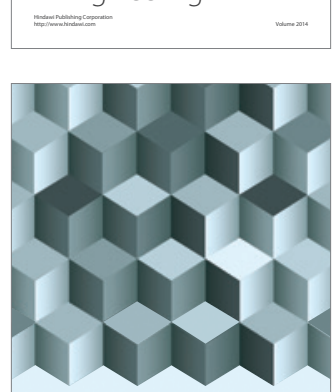

Journal of

Function Spaces
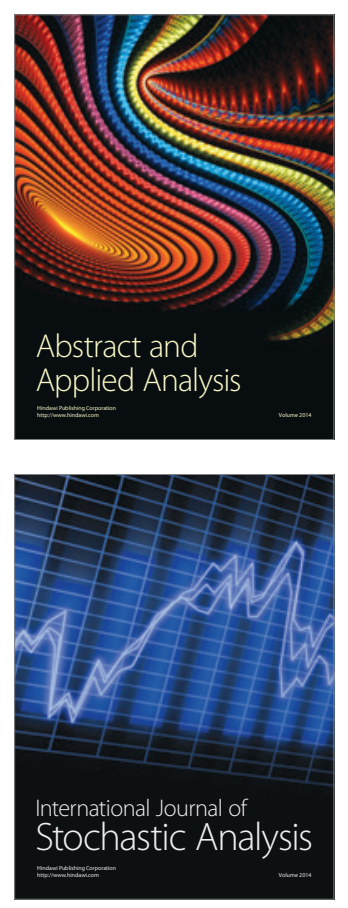

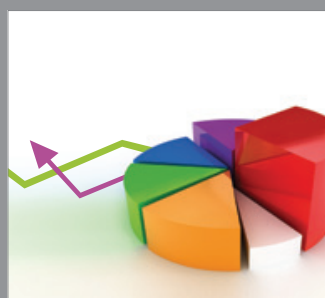

ournal of

Probability and Statistics

Promensencen
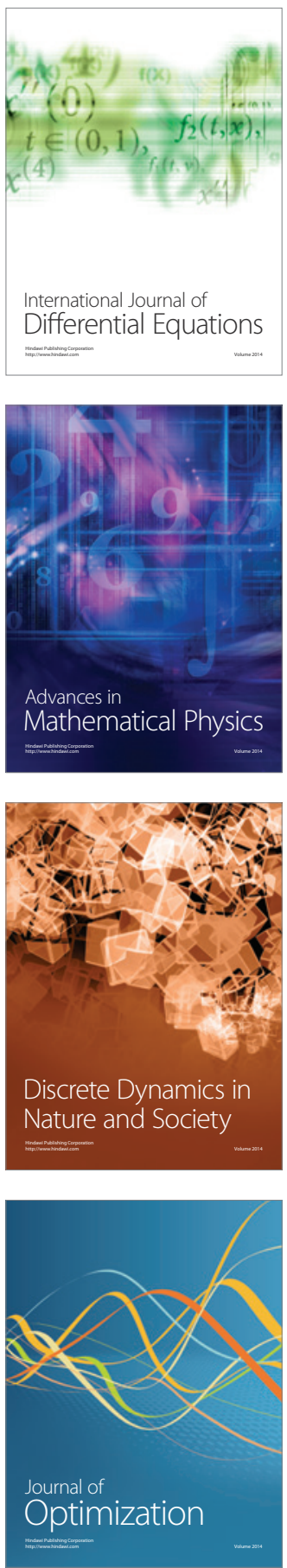